\title{
Type 2 diabetes mellitus and sepsis: state of the art, certainties and missing evidence
}

\author{
Elisa Costantini ${ }^{1,2} \cdot$ Massimiliano Carlin ${ }^{1,2} \cdot$ Massimo Porta ${ }^{1,2} \cdot$ Maria Felice Brizzi $^{1,2}$ (1)
}

Received: 1 March 2021 / Accepted: 22 April 2021 / Published online: 10 May 2021

(c) The Author(s) 2021

\begin{abstract}
Diabetes and sepsis are important causes of morbidity and mortality worldwide, and diabetic patients represent the largest population experiencing post-sepsis complications and rising mortality. Dysregulated immune pathways commonly found in both sepsis and diabetes contribute to worsen the host response in diabetic patients with sepsis. The impact of diabetes on mortality from sepsis is still controversial. Whereas a substantial proportion of severe infections can be attributed to poor glycemic control, treatment with insulin, metformin and thiazolidinediones may be associated with lower incidence and mortality for sepsis. It has been suggested that chronic exposure to high glucose might enhance immune adaptation, leading to reduced mortality rate in septic diabetic patients. On the other hand, higher risk of acute kidney injury has been extensively documented and a suggested lower risk of acute respiratory distress syndrome has been recently questioned. Additional investigations are ongoing to confirm the protective role of some anti-diabetic treatments, the occurrence of acute organ dysfunction, and the risk/benefit of less stringent glycemic control in diabetic patients experiencing sepsis. Based on a MEDLINE/PubMed search from inception to December 31, 2020, the aim of this review is therefore to summarize the strengths and weaknesses of current knowledge on the interplay between diabetes and sepsis.
\end{abstract}

Keywords Type 2 diabetes mellitus · Sepsis · Immune dysfunction · Glycemic control · Organ dysfunction · Mortality

\section{Introduction}

Sepsis is defined as a "life-threatening organ dysfunction caused by dysregulated host response to an infection", and represents a leading cause of death worldwide, with a mortality rate $>10 \%$ [1]. In 2017, almost 50 million incident cases of sepsis were estimated worldwide and 11 million sepsis-related deaths were reported, representing nearly $20 \%$ of all global deaths [2]. Septic shock is highly prevalent in the general population, occurring in the $8-10 \%$ of Intensive Care Unit (ICU) patients, with a high mortality rate (almost 40\%) [3]. The expanding elderly population suffering from extensive comorbidity burden, physiological frailty

Managed by Antonio Secchi .

Maria Felice Brizzi

mariafelice.brizzi@unito.it

1 Department of Medical Sciences, University of Turin, Corso Dogliotti 14, 10126 Turin, Italy

2 Azienda Ospedaliera Universitaria Città Della Salute E Della Scienza, Turin, Italy and immune senescence [4] leads to predict an increased mortality rate for sepsis over the next couple of decades [5].

With the rising globalization of Western diet and lifestyle, the incidence of Type 2 Diabetes Mellitus (T2D) is increasing and its prevalence is expected to exceed 700 million worldwide in the near future, reaching pandemic proportions [6]. T2D and diabetes-related complications are also a leading cause of hospitalization, disability and mortality [7, 8].

Although still under debate [9-11], several lines of evidence indicate that diabetic patients have an increased risk of infection [9, 12-17], and a 2 to 6 times higher risk of sepsis compared to the age-matched non-diabetic people $[12,17])$, and higher sepsis-related morbidity and mortality compared to non-diabetic individuals [12, 15, 18, 19]. Diabetic patients are also likely to have higher rates of colonization by resistant pathogens, including methicillin-resistant Staphylococcus aureus, than non-diabetics [20]. These considerations support the finding that diabetes is an increasingly common comorbidity among septic patients [21, 22]. As a matter of fact, during a 25-year study period (1979-2003), sepsis occurred in 12.5 million of 930 million acute-care hospitalizations, and diabetes was reported in $17 \%$ of cases [21]. 
Moreover, diabetic patients account for the largest population experiencing post-sepsis complications and rising mortality [15].

Despite current improvements in diagnosis and treatment options, diabetes and sepsis remain common, costly and lethal worldwide $[3,11,14]$. This work aims at reviewing the current state of knowledge about: (1) the impact of diabetes and sepsis on the immune system, (2) the influence of diabetes on the risk of sepsis and its outcomes, and (3) the optimal target for blood glucose control during sepsis in patients with diabetes.

A MEDLINE/PubMed search was conducted from inception to December 31, 2020, using the MeSH terms Diabetes mellitus AND Sepsis AND the following: Immune system processes, Glycated hemoglobin, Insulin, Hypoglycemic agents, Metformin, Sulphonylurea compounds, Thiazolidinediones, Incretin, Multiple-organ dysfunction syndrome, Lung injury, Acute respiratory distress syndrome, Acute kidney failure, Blood glucose, Mortality.

All types of publications and articles related to human studies were initially included. Out of 583 records retrieved through the initial database search, 425 remained after removing duplicates. After manual assessment based on title/abstract, 150 remained for full-text assessment for eligibility. Articles without full text or not written in English, case reports and studies involving generically critically ill patients or patients with specific infective focus were excluded. Based on these exclusion criteria, 92 records were excluded, while 58 articles remained. An additional 46 records were identified by manual search among the references cited in these records and further assessed for eligibility according to the above-mentioned criteria, leading to exclude 27 and include 19.

Finally, 77 studies were included in the qualitative analysis (Fig. 1).

\section{Interactions between type $\mathbf{2}$ diabetes and sepsis}

T2D is a complex clinical syndrome characterized by persistent hyperglycemia, associated with decreased insulin secretion and sensitivity [13]. Several metabolic abnormalities, including inflammation and insulin resistance driven by both chronic and stress-induced hyperglycemia, and T2Drelated obesity and dyslipidemia, additionally worsen the host response against infections.

Also, sepsis exerts a global impact on the immune system, impairing the lifespan, generation and function of innate and adaptive immune cells and leading to perturbation of the immune homeostasis [23].

Currently, the molecular network that cooperates to worsen clinical outcomes in patients with T2D and sepsis remains uncertain [15]. Figure 2 summarizes the current

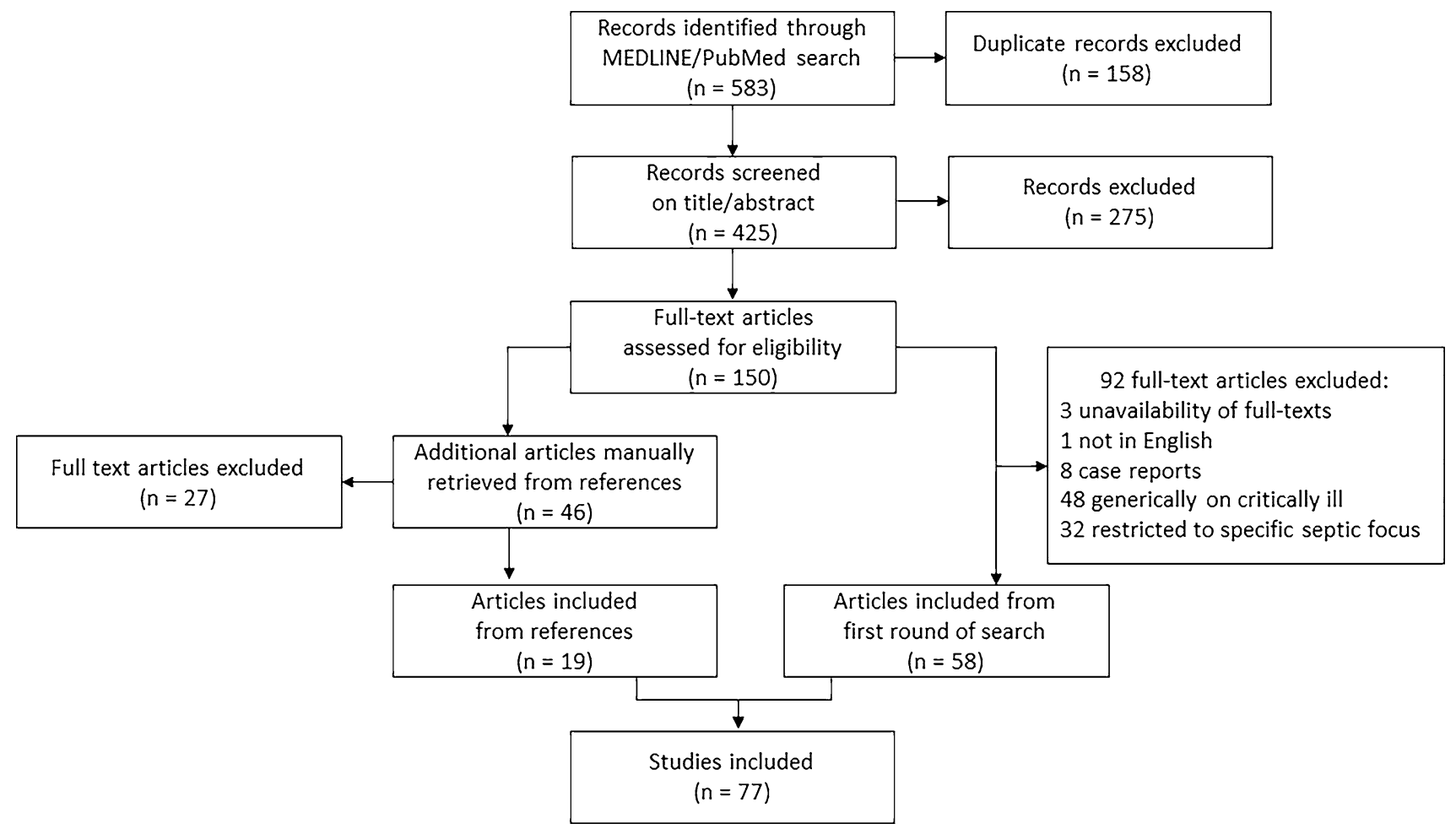

Fig. 1 Study flow diagram 


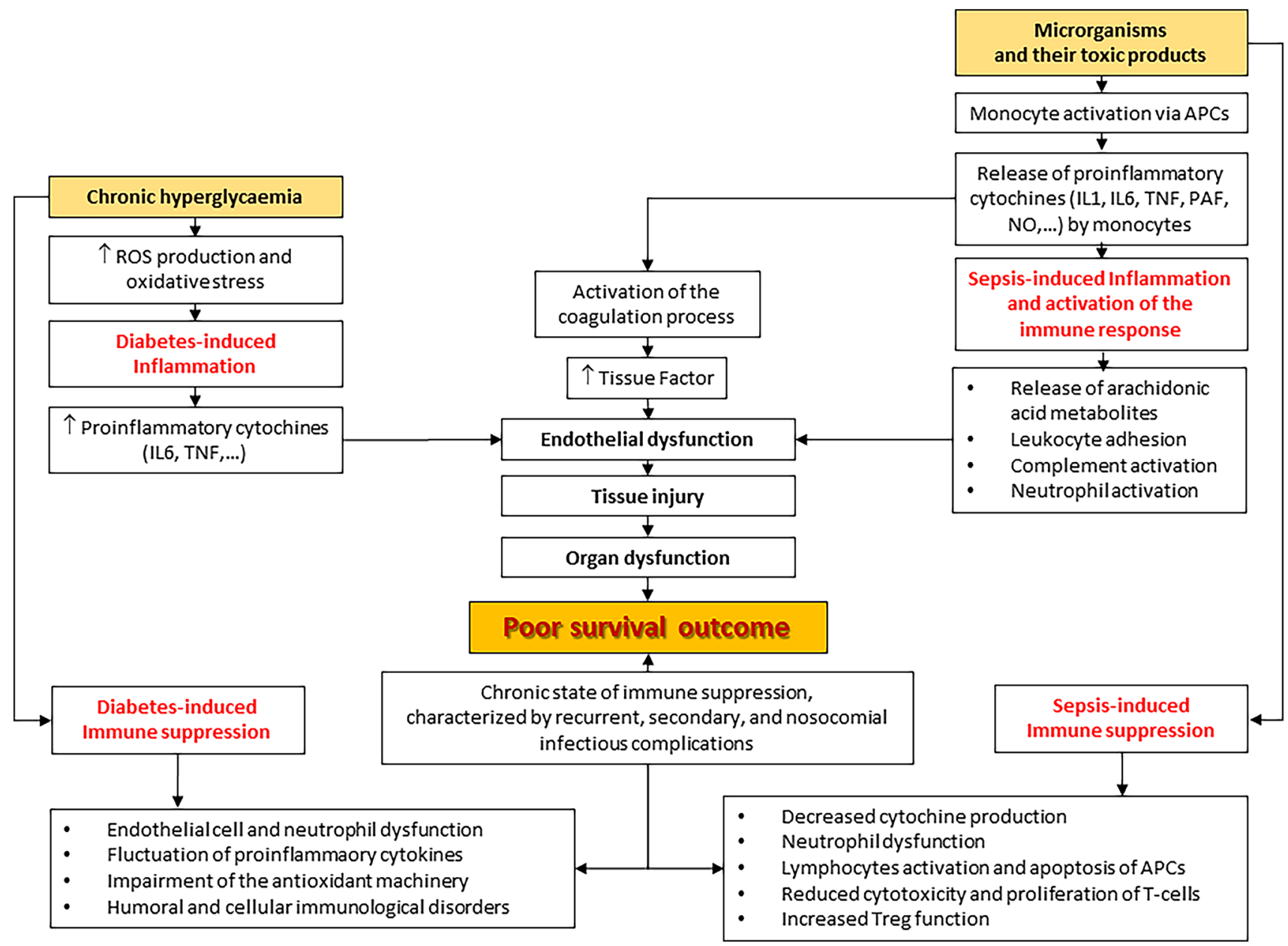

Fig. 2 Interactions between diabetes and sepsis in inflammation and on the immune system ( adapted from Tiwari et al. [13]). Both T2Drelated chronic hyperglycemia and toxic products released by invading microorganisms during sepsis contribute to increase inflammatory response [13]. It is generally accepted that the chronic and indolent inflammation induced by T2D and obesity differs from the acute inflammatory response caused by sepsis [77]. However, Frydrych et al. [15] outlined the impairment of several inflammatory responses in both T2D and sepsis (data not shown in the Figure), including: a increased levels of complement proteins (which are defective in T2D) driving systemic inflammation, organ failure and mortality; b mitochondrial dysfunction and redox imbalance as relevant mediators of disease progression; c impaired calcium homeostasis promoting elevated inflammatory responses, cellular dysfunction and toxicity. The increase in pro-inflammatory cytokines, induced by both T2D and sepsis, and the activation of the immune system due to sepsis are responsible for the endothelial dysfunction carrying the organ dysfunction characteristic of sepsis and accountable for poor outcome [13]. Additionally, functional neutrophil defects and deranged recruitment into sites of infection are commonly found not only in T2D but also in sepsis. Apoptosis of both lymphocytes and antigen-presenting cells (APCs) is a hallmark of septic-mediated immune suppression, whereas endothelial cell dysfunction, fluctuation of pro-inflammatory cytokines and the impairment of both the antioxidant machinery and humoral immunity are linked to TD2 [15]. Thus, in diabetic patients surviving from sepsis, the coexistence of the sepsis-induced immune activation over-described and such immune suppression related to both $\mathrm{T} 2 \mathrm{D}$ and sepsis weakens the immune response contributing to create a chronic immune suppression leading to further infective complications and poor long-term survival [15] knowledge on the mechanisms of sepsis and the effects of chronic hyperglycemia, both impacting on the immune system and translating into poor patient outcome $[13,15]$.

\section{Premorbid modifiers of the risk of sepsis}

\section{Long-term glycemic control and the risk of sepsis}

Glycated hemoglobin (HbA1c), term used to describe " $a$ series of stable minor hemoglobin components formed 
slowly and nonenzymatically from hemoglobin and glucose", is the most widely used marker of long-term glucoregulation and represents a risk mark for the development of diabetes complications [11, 24]. In hyperglycemic sepsis, it allows to distinguish non-diabetic individuals experiencing stress hyperglycemia from patients with previously undiagnosed diabetes and, comparing actual blood glucose values with the $\mathrm{HbA} 1 \mathrm{c}$-estimated average levels at preadmission, to identify stress-induced glycemic deterioration in patients with preadmission diagnosis of diabetes [25].

Only a few studies have investigated the relationship between glycemic control and infectious diseases [26, 27]. A recent review of higher-quality population-based epidemiological studies [26] have reported an association between high $\mathrm{HbAlc}(>7-8 \%$ or $>53-64 \mathrm{mmol} / \mathrm{mol})$ and a $1.5-3.5-$ fold increased risk of infection in diabetic patients. However, these studies are still debated, since their statistical power and controls for confounders are missed.

A further large-size retrospective cohort study [27] on more than 150,000 patients, among whom approximately 85,000 were diabetics (mostly T2D), confirmed a powerful association between poor glycemic control and high risks of serious infections (not just sepsis). Specifically, diabetic patients showed greater hospitalization risks for infections compared to non-diabetics, regardless of glycemic control (sepsis rates were elevated even among patients with $\mathrm{HbA} 1 \mathrm{c}<6 \%$ or $42 \mathrm{mmol} / \mathrm{mol}$ ). Nevertheless, for several infections, an association trend was found between increasing $\mathrm{HbA1c}$ level and the risk of infection. Within diabetic patients, a poor metabolic control was associated with a threefold risk of hospitalization. Overall, $15.7 \%$ of infectionrelated deaths, $16.5 \%$ of infection-related hospitalizations, $6.8 \%$ of infections requiring a prescription, and up to $20 \%$ of sepsis cases, have been attributed to $\mathrm{HbAlc}$ value different from $6-7 \%(42-53 \mathrm{mmol} / \mathrm{mol})$. In detail, the incidence rate ratio for sepsis ranged from 1.2 (for $\mathrm{HbA} 1 \mathrm{c} \geq 7 \%$ or $53 \mathrm{mmol} / \mathrm{mol}$ ) to 3.64 (for $\mathrm{HbA} 1 \mathrm{c} \geq 11 \%$ or $97 \mathrm{mmol} / \mathrm{mol}$ ). Interestingly, even a tight metabolic control $(\mathrm{HbA} 1 \mathrm{c}<6 \%$ or $42 \mathrm{mmol} / \mathrm{mol}$ ) was associated with an increased risk of infections in the older population, among whom the infectious risk and poor outcomes were found globally higher. The authors hypothesized for these patients that a less stringent glycemic control (up to $\mathrm{HbAlc} 8 \%$; $64 \mathrm{mmol} / \mathrm{mol}$ ) may be beneficial, while a tighter control would be associated with additional risks [27].

Thus, although evidence suggests that a better glycemic control might reduce the risk of infections, further trials including older patients, people with a poor metabolic control, and whit a history of significant infectious disease are required [26, 27].

\section{Impact of insulin and other anti-diabetic medications on the incidence and mortality for sepsis}

Immunomodulatory effects of both insulin and non-insulin glucose-lowering agents have been extensively documented, and their beneficial impact in diabetic patients with sepsis has been suggested [9, 11, 28-32].

Insulin may protect against over-activation of the immune system by preventing the adverse effects on immune functions related to high blood glucose and exerting direct and indirect anti-inflammatory effects $[9,28]$. However, two large-size observational studies failed to reveal differences in mortality attributable to previous insulin treatment. A first report on critically ill subjects (among whom 7\% with previously diagnosed insulin-treated diabetes) [33] revealed that formerly insulin-treated diabetic individuals were more severely ill, however, they did not display an increased mortality rate. In a further prospective observational study [34], including ICU septic patients with and without diabetes (the first either insulin- and non-insulin treated), the disease progression and mortality for sepsis in diabetic patients was similar regardless of insulin treatment.

Some non-insulin glucose-lowering agents have been associated with several immune-modulating effects in preclinical studies. Specifically, metformin may exert important pleiotropic effects, involving the regulation of lactate metabolism and AMPK activation, and produce anti-inflammatory, anti-endotoxemic, vasoactive and antimicrobial actions [31]. Thiazolidinediones (TZD) increase neutrophil migration, suggesting potential benefits in the modulation of the inflammatory response and in the outcome of septic patients [11]. An anti-inflammatory action has been shown and an immunomodulatory effect has been hypothesized also for incretin hormones, since they are involved in inflammatory response $[30,32,35]$. Preclinical models of sepsis have demonstrated that incretin-based therapies decrease immune cell activation, inhibit pro-inflammatory cytokine release and reduce organ dysfunction and mortality [32]. Although incretinbased therapies have not yet been tested in clinical trials of sepsis, it has been hypothesized that both incretin-mimetics [32] and DPP4 inhibitors [35] may exert positive pleiotropic effects on both inflammation and immunomodulation. On the contrary, insulin secretagogue-mediated off-target effects driven by the inhibition of the adenosine triphosphate-sensitive potassium channel in $\beta$ cells were found to impair the immune response against invading pathogens in preclinical studies [29].

A large nested case-control study analyzing the impact of current treatment with non-insulin agents on the incidence of sepsis [29] demonstrated that metformin may confer a persistent benefit on the rate of hospitalization for sepsis. TZD administration was also inversely associated with the 
occurrence of sepsis, unlike meglitinide. Treatment with sulfonylureas and DPP4 inhibitors is not associated with altered incidence of sepsis. In a recent meta-analysis, also Liang et al. [31] linked metformin treatment with reduced mortality in diabetic patients with sepsis. Nevertheless, although of interest, the reliability of this observation is limited by the relatively small sample size. A more recent, larger population-based cohort study [36] reported that metformin treatment is not significantly associated with the risk for sepsis nor with 30-day mortality for sepsis in diabetic patients. Although some small clinical trials in critically ill patients have suggested potential benefits in glycemic control using incretin infusion, these studies included mixed populations and had limited power [32].

Thus, an association between preadmission treatment with insulin or non-insulin glucose-lowering agents and the risk and outcome of sepsis remains controversial. The degree of glycemic control, rather than the anti-diabetic therapies, could explain the risk and mortality for sepsis. As a matter of fact, in a small observational study [37], HbA1c has been proved an independent prognostic factor for hospital mortality and time of hospitalization for diabetic septic patients, while no difference in the outcomes were found related to prior anti-diabetic treatments.

Further, clinical trials specifically investigating the potential benefits of anti-diabetic medications in septic cohorts are required.

\section{Optimal blood glucose control during sepsis}

Progression of sepsis is associated with changes in insulin and cortisol circulating levels, resulting in significant glucose perturbations, organ damage and activation of the immune system [38]. Besides the well-known stressinduced hyperglycemia, hypoglycemia may also reflects a pathological acute stress response. Indeed, hypoglycemia is commonly associated with sepsis and considered an epiphenomenon of severe organ dysfunction preceding death. Although the mechanisms and relationships between hypoglycemia and the severity of the disease in septic patients are still debated, the role of inflammatory cytokines has been proposed [39].

In critical settings, derangement of glycemic control is associated with more severe disease and poorer prognosis [39-41]. However, diabetes may modulate the relationship between dysglycemia and mortality in sepsis [40]. Indeed, the risk of mortality associated with hyperglycemia is lower in diabetic than non-diabetic patients [42] and is not influenced by hypoglycemia [39] or glycemic variability [43].

Despite strong recommendations for early insulin administration, how to monitor and treat stress-induced hyperglycemia remains under debate [41, 44].
Several large-size trials have investigated the optimal acute blood glucose control in critically ill patients, including septic ones [22, 45-47]. However, only a few small studies were restricted to septic patients [48, 49], and none specifically targeted diabetic patients. Table 1 reports the main clinical trials evaluating the impact of different targets of acute glycemic control in critically ill and septic patients. Van den Berghe et al. [45] first evaluated patients admitted to surgical Intensive Care Units (ICU) who were randomly assigned to receive intensive insulin therapy (blood glucose target $80-110 \mathrm{mg} / \mathrm{dl}$ ) or conventional therapy (target $180-200 \mathrm{mg} / \mathrm{dl}$ ). Although the number of septic patients was not reported at baseline, intensive insulin therapy reduced episodes of nosocomial septicaemia of about $46 \%$ and the proportion of patients requiring prolonged antibiotic therapy. Specifically, a tight glucose control (TGC, i.e., blood glucose levels $<110 \mathrm{mg} / \mathrm{dl}$ ) was associated with lower morbidity and mortality rates (with a $43 \%$ relative risk reduction of ICU mortality). However, this result relies of the benefit obtained in the subgroup of those patients staying in ICU for more than 5 days and in cardiac surgical patients (accounting for the majority of the study population) who previously received intravenous glucose load for nutritional purpose. In a subsequent study in medical ICU patients, the same group [46] failed to confirm a benefit on mortality in the overall population, since demonstrated that TGC prevents morbidity in all patients, but reduces mortality only in those staying in the ICU for at least 3 days. Moreover, concerns raised on the high rates of hypoglycemic events (more than sixfold higher than the previous study) in this subgroup of patients. A posthoc analysis [50] of pooled data from the two Leuven studies $[45,46]$ further confirmed that TGC carried a significantly higher risk of hypoglycemia (which occurred in $11.3 \%$ of patients on TGC vs. $1.8 \%$ of those on conventional insulin therapy, $p<0.0001)$. However, even if hypoglycemia was not associated with early deaths and/or neurological sequelae, a higher risk of death was reported. Such pooled data finally revealed that TGC significantly reduced morbidity and mortality in mixed medical/surgical ICU (particularly in patients staying in ICU at least 3 days). In addition it was reported that all patient subgroups, including those admitted for sepsis, benefit from TGC. Only for diabetic patients, no survival benefit was reported. A rapid normalization of blood glucose levels rather than hypoglycemic events has been proposed to explain the lack of TGC benefit in diabetic patients.

However, further studies failed to confirm these benefits from TGC [22, 46-48], although differences in study design, selection of patients, nutritional support, targeted glucose range and blood glucose measurements make the comparison challenging [41]. As a matter of fact, a further trial specifically involving patients with severe sepsis [48] not only failed to demonstrate a benefit on mortality from TGC, in both diabetic and non-diabetic patients, but was early 


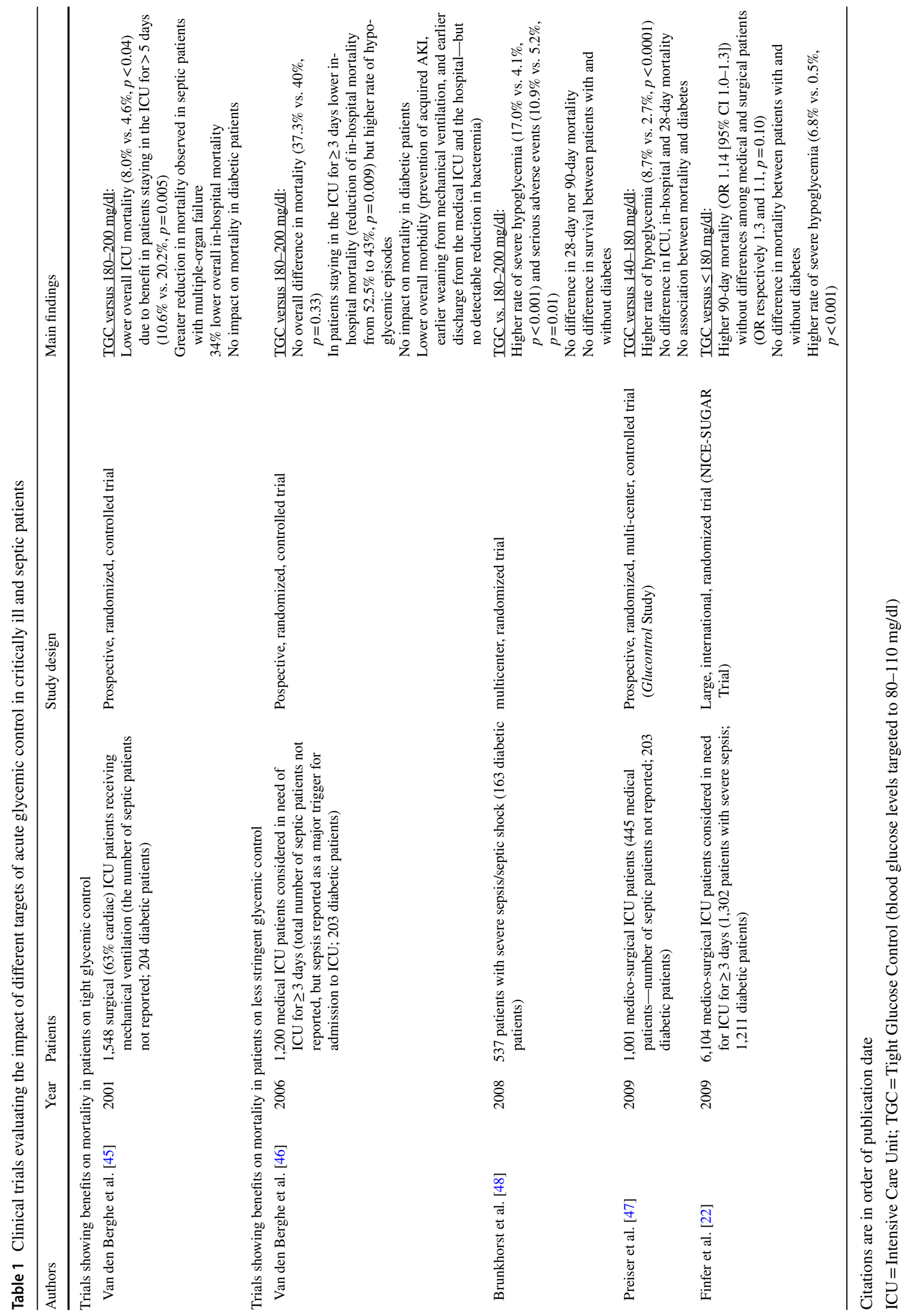


stopped for safety reasons (e.g., a significantly increased rate of severe hypoglycemic events). Two further large-scale trials including mixed populations of medical and surgical patients, the Glucontrol study [47] and the Normoglycemia in Intensive Care Evaluation-Survival Using Glucose Algorithm Regulation (NICE-SUGAR) Trial [22], reported higher rates of hypoglycemia in the TGC group. The former, prematurely stopped for the high rate of unintended protocol violations, did not find differences in mortality from TGC, while the second one revealed that a less stringent glycemic control translates into lower mortality rate, regardless of diabetes. Finally, a recent meta-analysis by Yamada et al. [51] confirmed the absence of clinical benefits of a stringent glycemic control in term of mortality, while reporting an increased rate of hypoglycemia in both diabetic and non-diabetic patients on TGC compared to patients on mild $(140-180 \mathrm{mg} / \mathrm{dl})$ and very mild control (180-220 mg).

The U-shaped curve describing the relationship between glycemic control and mortality (patients with low and high glucose levels have worse outcomes than those in the normal/moderate range) suggests that moderately elevated glycemic level may represent the ideal target in diabetic patients $[9,15,52]$. However, whether this effect was actually due to such glucose levels or to confounding variables driving hypoglycemia and poor outcome is still a matter of debate [52]. Additionally, the relationship between longer time in blood glucose range 70 to $140 \mathrm{mg} / \mathrm{dl}$ and lower mortality rate, clearly described in non-diabetic patients, is missing in diabetics [53].

Current guidelines recommend to treat hyperglycemia in critical patients to a target of $140-180 \mathrm{mg} / \mathrm{dL}$, regardless of the presence of previously known diabetes [54,55]. The need for specific targets of glycemic control in diabetic patients has been postulated [44, 56, 57], and some studies have suggested that less stringent glycemic control (e.g., targeting blood glucose levels at $180-250 \mathrm{mg} / \mathrm{dL}$ ) may be beneficial in critical patients with premorbid chronic hyperglycemia (e.g., HbA1c level $>7 \%$ or $>53 \mathrm{mmol} / \mathrm{mmol}$ ). However, concerns (including increased risk of infection, glycosuria and polyneuropathy) have raised against such permissive glucose levels in critically ill diabetic patients [58]. Based on these observations, Egi et al. [58] proposed to adopt a uniform blood glucose target for patients with and without diabetes $(\leq 180 \mathrm{mg} / \mathrm{dL})$, at least until the randomized control LUCID trial (Liberal GlUcose Control in Critically Ill Patients with Preexisting Type 2 Diabetes trial) [59] will inform on the risks and benefits of more liberal glucose control strategies.

Finally, a role was suggested for closed-loop glucose control systems and immunomodulatory treatment options, to avoid hypoglycemia during insulin therapy and to control the rise in circulating cytokine levels in diabetic patients with severe sepsis and septic shock [60].

\section{Acute organ dysfunction during sepsis}

The occurrence of organ dysfunction in diabetic patients with sepsis was first evaluated in a cohort of 12.5 million people admitted to hospital for sepsis between 1979 and 2003, among whom over 2 million had diabetes [21]. The study revealed that diabetic patients were less likely to develop acute respiratory failure ( $9 \%$ versus $14 \%, p<0.05$ ), regardless of the source of infection, but more likely to develop acute renal failure $(13 \%$ versus $7 \%, p<0.05)$ than non-diabetic ones. No differences were found in dysfunction of other organs (cardiovascular failure occurred in the $4 \%$ of the overall population, while hepatic, hematological, metabolic and central nervous system dysfunction globally occurred in the 6\%).

In both sepsis and chronic hyperglycemia, injury of the glycocalyx, due to generation of reactive oxygen species and inflammatory mediators, impacts the microcirculation and leads to organ damage [61]. The coexistence of diabetes and severe sepsis additionally compromises red blood cell deformability, worsens the microcirculation and hastens the progression of organ dysfunction [62].

A recent retrospective observational study [61] supports the link between premorbid chronic hyperglycemia and progression to organ dysfunction in septic patients. The authors demonstrated that, in septic patients admitted to the ICU, HbA1c values $\geq 6.5 \%$ ( $\geq 47.5 \mathrm{mmol} / \mathrm{mmol}$ ) were independently associated with the progression of liver and kidney failure within $72 \mathrm{~h}$, and with ICU mortality. Only, a positive trend for the progression of lung and cardiac dysfunction and clotting disorders was reported.

Unlike lower risk of acute respiratory dysfunction [14], higher risk of acute kidney injury in diabetic patients was confirmed in a large nationwide retrospective study [63] and in a recent meta-analysis [14].

\section{Acute respiratory failure}

Acute Lung Injury (ALI) and Acute Respiratory Distress Syndrome (ARDS) are common life-threatening conditions in critically ill patients. A protective role of diabetes against the development of ALI/ARDS has been extensively documented in different cohorts of critically ill and septic patients [28, 63-67]. However, such protection has recently been questioned, since a meta-analysis by Wang et al. [14] demonstrated a similar incidence of respiratory dysfunction in diabetic and non-diabetic septic patients.

Moreover, the potential mechanisms for such presumptive protective effect are still unclear [21, 28, 63, 66, 67]. Among the proposed mechanisms the impaired neutrophil function and altered neutrophil-endothelial interaction [68], the immunomodulatory effects mediated by hyperglycemia 
and resulting in a impaired response against endotoxinmediated injury [28, 68], as well as the presence of obesity and dyslipidemia-related metabolic effects [28] are included. Finally, some potential protective effects may result from extensive and earlier medical care and the anti-inflammatory properties of anti-diabetic medications, including insulin, TZD and metformin [21]. Similarly, whether and how diabetes may conversely contribute to increased incidence of other organ dysfunction in sepsis, such as renal failure, is still unclear [66].

\section{Acute kidney injury (AKI)}

AKI develops in one-fourth of all septic patients and half of those with bacteremia or shock [69]. In diabetic patients, observational studies reported an incidence of AKI ranging from $27 \%$ [63] to $73 \%$ [70].

Although diabetes is an established risk factor for both AKI [21, 33] and sepsis [9, 12-16], and reported as an independent risk factor for persistent renal dysfunction in ICU septic patients developing AKI [70], the impact of diabetes in increasing the risk of AKI is still debated.

As a matter of fact, in a prospective single-center ICU study [71], elevated serum creatinine on the first day was associated with the occurrence of AKI in patients with severe sepsis, though not in diabetic patients. In a further large cross-sectional multicenter study involving patients with severe sepsis or septic shock, diabetes resulted not associated with the occurrence of AKI or the need for renal replacement therapy [70]. Despite these findings and the wide heterogeneity of data related to the incidence of AKI in diabetic septic patients, the above-mentioned meta-analysis [14] confirmed that the incidence of AKI is definitely increased (over 50\%) in diabetic compared to non-diabetic septic patients.

\section{Mortality from sepsis}

Increased susceptibility to infection and sepsis in diabetes is extensively documented [9,12-17], but equivocal results from epidemiological studies pose doubts on the association between diabetes and increased risk of infection-related morbidity and mortality [9-11]. Different study populations (including lack of stratification into Type 1 and Type 2 Diabetes, different adjustments for comorbidities, sepsis etiology and disease severity), drug administration regimens to control blood glucose and methods to measure outcomes have been proposed to explain this heterogeneity $[10,11]$.

Table 2 reports recent clinical studies investigating the association between diabetes and mortality for sepsis. Among them, a large-size observational study [72] demonstrated that diabetic patients experienced an increased mortality from infectious diseases (persisting even after adjustment for comorbidities) and a twofold increased risk of mortality for sepsis compared to the general population. Additionally, two large-size retrospective cohort studies [12, 19] found higher mortality rate related to infections in diabetic compared to non-diabetic patients, whereas others [33, $34,63,66,70,73,74]$ failed to demonstrate such association, and Esper et al. [21] even reported improved survival in diabetic patients.

Few observational studies have investigated the link between premorbid glycemic state and sepsis outcome, showing that $\mathrm{HbA} 1 \mathrm{c}$ levels at admission are in direct correlation with hospital mortality in diabetic patients with sepsis [37, 61].

The results by Tayek et al. [75], firstly reporting a global benefit on mortality from diabetic status, were confirmed in the meta-analysis by Wang et al. [14], which demonstrated that diabetes is not associated with adverse outcomes in patients with sepsis, while beneficial. As a matter of fact, some studies notably demonstrated an association between hyperglycemia and increased mortality in non-diabetic patients, unlike in diabetic patients, suggesting that acute hyperglycemia may drive different pathophysiologic effects in diabetic and non-diabetic patients. Nevertheless, whether the link between hyperglycemia and mortality in non-diabetics relies on hyperglycemia-induced toxic effects or is the hallmark of disease severity still remains to be clarified [9]. Although the mechanisms for such protective effect driven by diabetes remain uncertain, previous exposure to high glucose has been proposed to enhance immune adaptation and to induce benefits $[9,14,75]$. The role of inflammation has been also investigated in this context. In particular, Stegenga et al. [73] reported comparable cytokine levels and procoagulant responses in critical septic patients with and without preexisting diabetes, while a different study unveiled the presence of elevated levels of markers of endothelial cell activation in patients with diabetes and septic shock, compared to patients without diabetes [76]. Beneficial effects of insulin administration, prevention of acute lung injury, adaptation to previous oxidant stress and nutritional intake in obese patients with diabetes were also proposed as protective against sepsis [9].

\section{Conclusions}

Sepsis represents a rising cause of mortality worldwide and diabetes is a common and increasing comorbidity in septic patients. Although the higher risk of infection in diabetic patients is well documented, the impact of diabetes on the outcome of sepsis and the mechanisms underlying their interactions are still debated. 


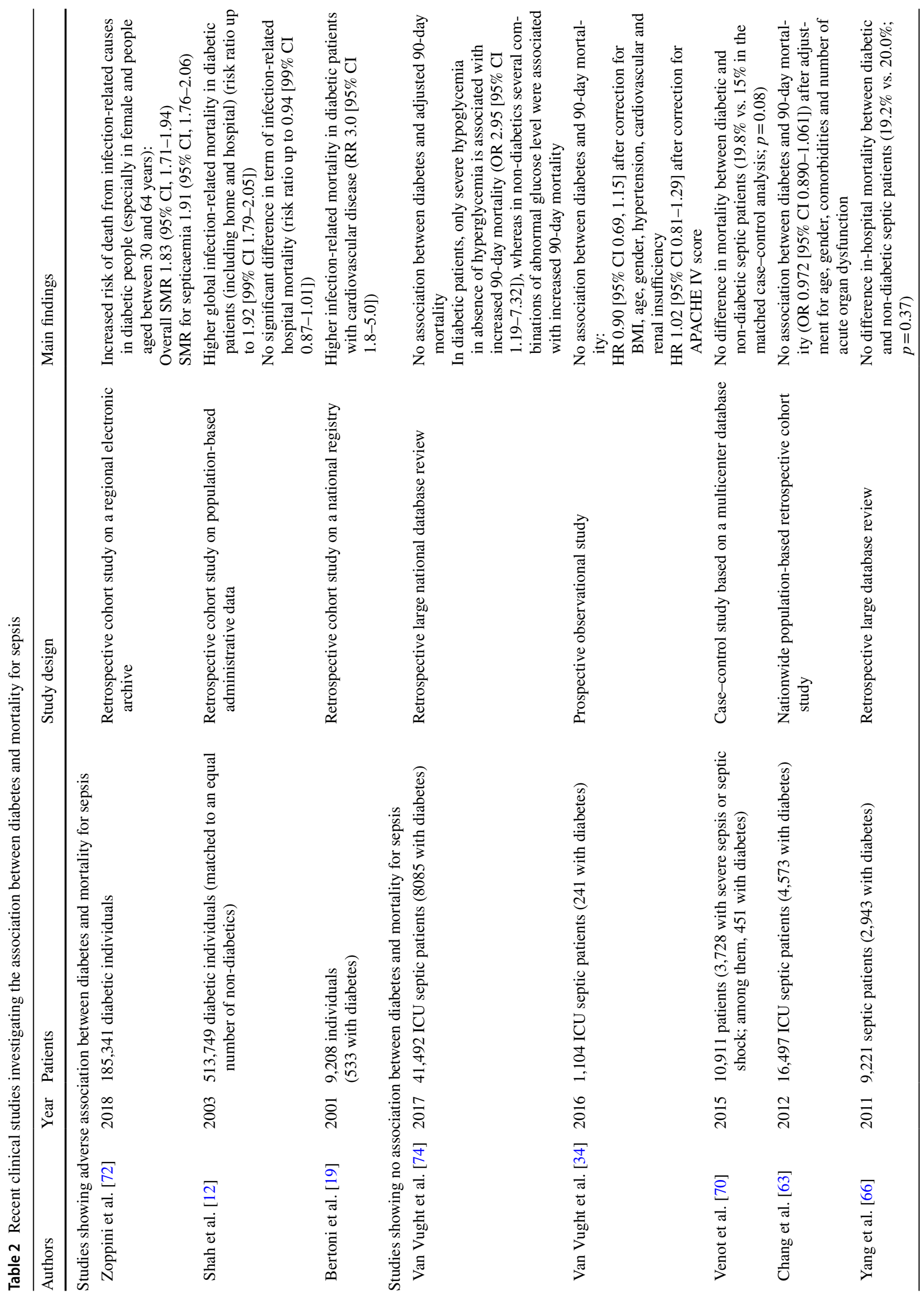


Critical issues that need clarifying include the impact of diabetes and sepsis on the immune system, the role of glycemic control and the potential protective role of anti-diabetic treatments, on the occurrence of sepsis and its outcome, including the risk of renal failure and acute respiratory dysfunction. Also, recommendations for glycemic targets during sepsis do not stand on firm grounds.

Further large-size prospective studies, randomized controlled trials whenever possible, specifically including diabetic patients with sepsis instead of generically critically ill or patients with specific infective focus, could clear some of these unsolved questions, including the risk/benefit balance of more liberal acute glycemic control.

Finally, interesting and challenging therapeutic options, including immunomodulatory approaches targeting the pathways activated in T2D and sepsis, are under investigation and may result in clinical benefits.

Funding Open access funding provided by Università degli Studi di Torino within the CRUI-CARE Agreement.

\section{Declarations}

Conflict of interest The authors have no competing interests to declare.

Ethical approval As this was a review study, no ethics approval wasrequired.

Informed consent Not applicable.

Open Access This article is licensed under a Creative Commons Attribution 4.0 International License, which permits use, sharing, adaptation, distribution and reproduction in any medium or format, as long as you give appropriate credit to the original author(s) and the source, provide a link to the Creative Commons licence, and indicate if changes were made. The images or other third party material in this article are included in the article's Creative Commons licence, unless indicated otherwise in a credit line to the material. If material is not included in the article's Creative Commons licence and your intended use is not permitted by statutory regulation or exceeds the permitted use, you will need to obtain permission directly from the copyright holder. To view a copy of this licence, visit http://creativecommons.org/licenses/by/4.0/.

\section{References}

1. Singer M, Deutschman CS, Seymour C et al (2016) The third international consensus definitions for sepsis and septic shock (sepsis-3). JAMA 315:801-810. https://doi.org/10.1001/jama. 2016.0287

2. Rudd KE, Johnson SC, Agesa KM et al (2020) Global, regional, and national sepsis incidence and mortality, 1990-2017: analysis for the global burden of disease study. Lancet 395:200-211. https://doi.org/10.1016/S0140-6736(19)32989-7

3. Vincent JL, Jones G, David S et al (2019) Frequency and mortality of septic shock in Europe and North America: a systematic review and meta-analysis. Crit Care 23:1-11. https://doi.org/10. 1186/s13054-019-2478-6 
4. Martin GS, Mannino DM, Moss M (2006) The effect of age on the development and outcome of adult sepsis. Crit Care Med 34:15-21. https://doi.org/10.1097/01.ccm.0000194535.82812.ba

5. Kahn JM, Le T, Angus DC et al (2015) The epidemiology of chronic critical illness in the United States. Crit Care Med 43:282-287. https://doi.org/10.1097/CCM.0000000000000710

6. Zimmet P, Alberti KGMM, Shaw J (2001) Global and societal implications of the diabetes epidemic. Nature 414:782-787. https://doi.org/10.1038/414782a

7. Bhutani J, Bhutani S (2014) Worldwide burden of diabetes. Indian J Endocrinol Metab 18:868-870. https://doi.org/10.4103/22308210.141388

8. Monge L, Gnavi R, Carnà $\mathrm{P}$ et al (2020) Incidence of hospitalization and mortality in patients with diabetic foot regardless of amputation: a population study. Acta Diabetol 57:221-228. https://doi.org/10.1007/s00592-019-01412-8

9. Schuetz P, Castro P, Shapiro NI (2011) Diabetes and sepsis: preclinical findings and clinical relevance. Diabetes Care 34:771778. https://doi.org/10.2337/dc10-1185

10. Koh GCKW, Peacock SJ, Van Der PT, Wiersinga WJ (2012) The impact of diabetes on the pathogenesis of sepsis. Eur J Clin Microbiol Infect Dis 31:379-388. https://doi.org/10.1007/ s10096-011-1337-4

11. Trevelin SC, Carlos D, Beretta M et al (2017) Diabetes mellitus and sepsis: a challenging association. Shock 47:276-287. https:// doi.org/10.1097/SHK.0000000000000778

12. Shah BR, Hux JE (2003) Quantifying the risk of infectious diseases for people with diabetes. Diabetes Care 26:510-513. https:// doi.org/10.2337/diacare.26.2.510

13. Tiwari S, Pratyush DD, Gahlot A, Singh SK (2011) Sepsis in diabetes: a bad duo. Diabetes Metab Syndr Clin Res Rev 5:222-227. https://doi.org/10.1016/j.dsx.2012.02.026

14. Wang Z, Ren J, Wang G et al (2017) Association between diabetes mellitus and outcomes of patients with sepsis: a meta-analysis. Med Sci Monit 23:3546-3555. https://doi.org/10.12659/MSM. 903144

15. Frydrych LM, Fattahi F, He K et al (2017) Diabetes and sepsis: risk, recurrence, and ruination. Front Endocrinol (Lausanne) 8:1-22. https://doi.org/10.3389/fendo.2017.00271

16. Muller LMAJ, Gorter KJ, Hak E et al (2005) Increased risk of common infections in patients with type 1 and type 2 diabetes mellitus. Clin Infect Dis 41:281-288. https://doi.org/10.1086/ 431587

17. Laupland KB, Gregson DB, Zygun DA et al (2004) Severe bloodstream infections: a population-based assessment. Crit Care Med 32:992-997. https://doi.org/10.1097/01.CCM.0000119424.31648. $1 \mathrm{E}$

18. Frydrych LM, Bian G, O'Lone DE et al (2018) Obesity and type 2 diabetes mellitus drive immune dysfunction, infection development, and sepsis mortality. J Leukoc Biol 104:525-534. https:// doi.org/10.1002/JLB.5VMR0118-021RR

19. Bertoni AG, Saydah S, Brancati FL (2001) Diabetes and the risk of infection-related mortality in the U.S. Diabetes Care 24:10441049. https://doi.org/10.2337/diacare.24.6.1044

20. Stacey HJ, Clements CS, Welburn SC, Jones JD (2019) The prevalence of methicillin-resistant Staphylococcus aureus among diabetic patients: a meta-analysis. Acta Diabetol 56:907-921. https:// doi.org/10.1007/s00592-019-01301-0

21. Esper AM, Moss M, Martin GS (2009) The effect of diabetes mellitus on organ dysfunction with sepsis: an epidemiological study. Crit Care 13:9-14. https://doi.org/10.1186/cc7717

22. Finfer A, Chittock D, Su S et al (2009) Intensive versus conventional glucose control in critically ill patients. N Engl J Med 360:1283-1297. https://doi.org/10.1056/NEJMoa0810625
23. Bosmann M, Ward PA (2013) The inflammatory response in sepsis. Trends Immunol 34:129-136. https://doi.org/10.1016/j.it. 2012.09.004

24. Goldstein DE, Little RR, Lorenz RA et al (2004) Tests of glycemia in diabetes. Diabetes Care 27:1761-1773. https://doi.org/10.2337/ diacare.27.7.1761

25. Braithwaite SS (2010) Through the eyes of the A1C: a call to reexamine stress hyperglycemia. Crit Care Med 38:717-719. https:// doi.org/10.1097/CCM.0b013e3181cc8c6a

26. Pearson-Stuttard J, Blundell S, Harris T et al (2016) Diabetes and infection: assessing the association with glycaemic control in population-based studies. Lancet Diabetes Endocrinol 4:148-158. https://doi.org/10.1016/S2213-8587(15)00379-4

27. Critchley JA, Carey IM, Harris T et al (2018) Glycemic control and risk of infections among people with type 1 or type 2 diabetes in a large primary care cohort study. Diabetes Care 41:2127-2135. https://doi.org/10.2337/dc18-0287

28. Honiden S, Gong M (2009) Diabetes, insulin, and development of acute lung injury. Crit Care Med 37:2455-2464. https://doi.org/ 10.1097/CCM.0b013e3181a0fea5

29. Shih CJ, Wu YL, Chao PW et al (2015) Association between use of oral anti-diabetic drugs and the risk of sepsis: a nested casecontrol study. Sci Rep 5:1-7. https://doi.org/10.1038/srep15260

30. Perl SH, Bloch O, Zelnic-Yuval D et al (2018) Sepsis-induced activation of endogenous GLP-1 system is enhanced in type 2 diabetes. Diabetes Metab Res Rev 34:1-8. https://doi.org/10.1002/ dmrr.2982

31. Liang H, Ding X, Li L et al (2019) Association of preadmission metformin use and mortality in patients with sepsis and diabetes mellitus: a systematic review and meta-analysis of cohort studies. Crit Care 23:1-9. https://doi.org/10.1186/s13054-019-2346-4

32. Shah FA, Mahmud H, Gallego-Martin T et al (2019) Therapeutic effects of endogenous incretin hormones and exogenous incretinbased medications in sepsis. J Clin Endocrinol Metab 104:5274 5284. https://doi.org/10.1210/jc.2019-00296

33. Vincent J, Preiser J, Sprung C et al (2010) Insulin-treated diabetes is not associated with increased mortality in critically ill patients. Crit Care Med 14:1-8. https://doi.org/10.1097/CCM.0000000000 002590

34. Van Vught LA, Scicluna BP, Hoogendijk AJ et al (2016) Association of diabetes and diabetes treatment with the host response in critically ill sepsis patients. Crit Care 20:1-15. https://doi.org/10. 1186/s13054-016-1429-8

35. Pantanetti P, Cangelosi G, Ambrosio G (2020) Potential role of incretins in diabetes and COVID-19 infection: a hypothesis worth exploring. Intern Emerg Med 15:779-782. https://doi.org/ $10.1007 / \mathrm{s} 11739-020-02389-\mathrm{x}$

36. Oh T, Song I (2020) Association between prior metformin therapy and sepsis in diabetes patients: a nationwide sample cohort study. J Anesth 34:358-366. https://doi.org/10.1007/s00540-020-02753-3

37. Gornik I, Gornik O, Gašparović V (2007) HbA1c is outcome predictor in diabetic patients with sepsis. Diabetes Res Clin Pract 77:120-125. https://doi.org/10.1016/j.diabres.2006.10.017

38. Maitra SR, Wojnar MM, Lang CH (2000) Alterations in tissue glucose uptake during the hyperglycemic and hypoglycemic phases of sepsis. Shock 13:379-385. https://doi.org/10.1097/ 00024382-200005000-00006

39. Kushimoto S, Abe T, Ogura $\mathrm{H}$ et al (2020) Impact of blood glucose abnormalities on outcomes and disease severity in patients with severe sepsis: an analysis from a multicenter, prospective survey of severe sepsis. PLoS ONE 15:1-15. https://doi.org/10. 1371/journal.pone.0229919

40. Krinsley JS, Egi M, Kiss A et al (2013) Diabetic status and the relation of the three domains of glycemic control to mortality in critically ill patients: an international multicenter cohort study. Crit Care 17:1-17. https://doi.org/10.1186/cc12547 
41. Stoudt K, Chawla S (2019) Don't sugar coat it: glycemic control in the ICU. J Intensive Care Med 34:889-896. https://doi.org/10. 1177/0885066618801748

42. Egi M, Bellomo R, Stachowski E et al (2008) Blood glucose concentration and outcome of critical illness: the impact of diabetes. Crit Care Med 36:2249-2255. https://doi.org/10.1097/CCM. Ob013e318181039a

43. Krinsley JS (2009) Glycemic variability and mortality in critically ill patients: the impact of diabetes. J Diabetes Sci Technol 3:1292-1301. https://doi.org/10.1177/193229680900300609

44. Abdelmalak BB, Lansang MC (2013) Revisiting tight glycemic control in perioperative and critically ill patients: when one size may not fit all. J Clin Anesth 25:499-507. https://doi.org/10. 1016/j.jclinane.2012.09.006

45. Van den Berghe G, Wouters P, Weekers F et al (2001) Intensive insulin therapy in critically ill patients. N Engl J Med 345:1359-1367

46. Van den Berghe G, Wilmer A, Hermans G et al (2006) Intensive insulin therapy in the medical ICU. N Engl J Med 354:449-461

47. Preiser JC, Devos P, Ruiz-Santana S et al (2009) A prospective randomised multi-centre controlled trial on tight glucose control by intensive insulin therapy in adult intensive care units: the glucontrol study. Intensive Care Med 35:1738-1748. https://doi.org/ 10.1007/s00134-009-1585-2

48. Brunkhorst FM, Engel C, Bloos F et al (2008) Intensive insulin therapy and pentastarch resuscitation in severe sepsis. N Engl $\mathrm{J}$ Med 358:125-139. https://doi.org/10.1177/175114370800900121

49. Song F, Zhong LJ, Han L et al (2014) Intensive insulin therapy for septic patients: a meta-analysis of randomized controlled trials. Biomed Res Int. https://doi.org/10.1155/2014/698265

50. Van den Berghe G, Wilmer A, Milants I et al (2006) Intensive insulin therapy in mixed medical/surgical intensive care unitsbenefit versus harm. Diabetes 55:3151-3159. https://doi.org/10. 2337/db06-0855

51. Yamada T, Shojima N, Noma H et al (2017) Glycemic control, mortality, and hypoglycemia in critically ill patients: a systematic review and network meta-analysis of randomized controlled trials. Intensive Care Med 43:1-15. https://doi.org/10.1007/ s00134-016-4523-0

52. Park JI, Bae E, Kim YL et al (2015) Glycemic control and mortality in diabetic patients undergoing dialysis focusing on the effects of age and dialysis type: a prospective cohort study in Korea. PLoS ONE 10:1-14. https://doi.org/10.1371/journal.pone.01360 85

53. Krinsley JS, Preiser JC (2015) Time in blood glucose range 70 to $140 \mathrm{mg} / \mathrm{dl}>80 \%$ is strongly associated with increased survival in non-diabetic critically ill adults. Crit Care 19:1-11. https://doi. org/10.1186/s13054-015-0908-7

54. Rhodes A, Evans LE, Alhazzani W et al (2017) Surviving sepsis campaign: International guidelines for management of sepsis and septic shock: 2016. Intensive Care Med 43:304-377. https://doi. org/10.1007/s00134-017-4683-6

55. American Diabete Association (2020) Standards of medical care in diabetes-2020. Diabetes Care 43:S195. https://doi.org/10. $2337 / \mathrm{dc} 20-\mathrm{S} 002$

56. Hirasawa H, Oda S, Nakamura M (2009) Blood glucose control in patients with severe sepsis and septic shock. World J Gastroenterol 15:4132-4136. https://doi.org/10.3748/wjg.15.4132

57. Egi M, Krinsley JS, Maurer P et al (2016) Pre-morbid glycemic control modifies the interaction between acute hypoglycemia and mortality. Intensive Care Med 42:562-571. https://doi.org/10. 1007/s00134-016-4216-8

58. Egi M (2018) Acute glycemic control in diabetics. How sweet is optimal? Con: just as sweet as in nondiabetic is better. J Intensive Care 6:1-3. https://doi.org/10.1186/s40560-018-0336-2
59. Poole AP, Finnis ME, Anstey J et al (2020) Study protocol and statistical analysis plan for the liberal glucose control in critically Ill patients with pre-existing type 2 diabetes (LUCID) trial. Crit care Resusc 22:133-141

60. Salinas PD, Mendez CE (2019) Glucose management technologies for the critically ill. J Diabetes Sci Technol 13:682-690. https:// doi.org/10.1177/1932296818822838

61. Lee YS, Min KH, Lee SY et al (2019) The value of glycated hemoglobin as predictor of organ dysfunction in patients with sepsis. PLoS ONE 14:1-13. https://doi.org/10.1371/JOURNAL. PONE.0216397

62. Moutzouri AG, Athanassiou GA, Dimitropoulou D et al (2008) Severe sepsis and diabetes mellitus have additive effects on red blood cell deformability. J Infect 57:147-151. https://doi.org/10. 1016/j.jinf.2008.04.004

63. Chang CW, Kok VC, Tseng TC et al (2012) Diabetic patients with severe sepsis admitted to intensive care Unit do not fare worse than non-diabetic patients: a nationwide population-based cohort study. PLoS ONE 7:1-10. https://doi.org/10.1371/journal.pone. 0050729

64. Moss M, Guidot D, Steinberg K et al (2000) Diabetic patients have a decreased incidence of acute respiratory distress syndrome. Crit Care Med 28:2187-2192. https://doi.org/10.1097/00003246200007000-00001

65. Gong MN, Thompson BT, Williams P et al (2005) Clinical predictors of and mortality in acute respiratory distress syndrome: potential role of red cell transfusion. Crit Care Med. https://doi. org/10.1097/01.CCM.0000165566.82925.14

66. Yang Y, Abdul Salam ZH, Ong BC, Yang KS (2011) Respiratory dysfunction in patients with sepsis: protective effect of diabetes mellitus. Am J Crit Care 20:e41-47. https://doi.org/10.4037/ajcc2 011391

67. Yu S, Christiani D, Thompson B et al (2013) Role of diabetes in the development of acute respiratory distress syndrome. Crit Care Med 41:2720-2732. https://doi.org/10.1097/CCM.0b013 e318298a2eb

68. Alexiewicz JM, Kumar D, Smogorzewski M et al (1995) Polymorphonuclear leukocytes in non-insulin-dependent diabetes mellitus: abnormalities in metabolism and function. Ann Intern Med 123:919-924. https://doi.org/10.7326/0003-4819-123-12-19951 2150-00004

69. Schrier RW, Wang W (2004) Acute renal failure and sepsis. N Engl J Med 351:159-169. https://doi.org/10.1056/nejmra032401

70. Venot M, Weis L, Clec'h C et al (2015) Acute kidney injury in severe sepsis and septic shock in patients with and without diabetes mellitus: a multicenter study. PLoS One 10:1-10. https://doi. org/10.1371/journal.pone.0127411

71. Yegenaga I, Hoste E, Van Biesen W et al (2004) Clinical characteristics of patients developing ARF due to sepsis/systemic inflammatory response ryndrome: results of a prospective study. Am J Kidney Dis 43:817-824. https://doi.org/10.1053/j.ajkd.2003.12. 045

72. Zoppini G, Fedeli U, Schievano E et al (2018) Mortality from infectious diseases in diabetes. Nutr Metab Cardiovasc Dis 28:444-450. https://doi.org/10.1016/j.numecd.2017.12.007

73. Stegenga ME, Vincent JL, Vail GM et al (2010) Diabetes does not alter mortality or hemostatic and inflammatory responses in patients with severe sepsis. Crit Care Med 38:539-545. https:// doi.org/10.1097/CCM.0b013e3181c02726

74. Van Vught LA, Holman R, De Jonge E et al (2017) Diabetes is not associated with increased 90-day mortality risk in critically ill patients with sepsis. Crit Care Med 45:e1026-e1035. https:// doi.org/10.1097/CCM.0000000000002590

75. Tayek CJ, Tayek JA (2012) Diabetes patients and non-diabetic patients intensive care unit and hospital mortality risks associated 
with sepsis. World J Diabetes 3:29-34. https://doi.org/10.4239/ wjd.v3.i2.29

76. Schuetz P, Yano K, Sorasaki M et al (2011) Influence of diabetes on endothelial cell response during sepsis. Diabetologia 54:9961003. https://doi.org/10.1007/s00125-011-2059-y

77. Goldfine AB, Fonseca V, Shoelson SE (2011) Therapeutic approaches to target inflammation in type 2 diabetes. Clin Chem 57:162-167. https://doi.org/10.1373/clinchem.2010.148833
Publisher's Note Springer Nature remains neutral with regard to jurisdictional claims in published maps and institutional affiliations. 